\title{
ОПИСАНИЕ ЛИНЕЙНОГО ЭФФЕКТА ПЕРРОНА ПРИ ПАРАМЕТРИЧЕСКИХ ВОЗМУЩЕНИЯХ, ЭКСПОНЕНЦИАЛЬНО УБЫВАЮЩИХ К НУЛЮ НА БЕСКОНЕЧНОСТИ
}

\author{
Е. А. Барабанов, В. В. Быков
}

\begin{abstract}
Пусть $\mathcal{M}_{n}$ - множество линейных дифференциальных систем порядка $n$ с непрерывными и ограниченными на временно́й полуоси $\mathbb{R}_{+}$коэффициентами, $n \geqslant 2$. Показатели Ляпунова системы $A \in \mathcal{M}_{n}$ обозначаются через $\lambda_{1}(A) \leqslant \ldots \leqslant \lambda_{n}(A)$, их спектр - через $\Lambda(A)=\left(\lambda_{1}(A), \ldots, \lambda_{n}(A)\right)$ и ее индекс экспоненциальной устойчивости (размерность линейного подпространства решений с отрицательными характеристическими показателями) - через es $(A)$. Для системы $A \in \mathcal{M}_{n}$ и метрического пространства $M$ рассматривается класс $\mathcal{E}_{n}[A](M)$ непрерывных по совокупности переменных $(n \times n)$-матричнозначных функций $Q: \mathbb{R}_{+} \times M \rightarrow \mathbb{R}^{n \times n}$, удовлетворяющих оценке $\|Q(t, \mu)\| \leqslant C_{Q} \exp \left(-\sigma_{Q} t\right)$ для всех $(t, \mu) \in \mathbb{R}_{+} \times M$, где $C_{Q}$ и $\sigma_{Q}$ - положительные постоянные (свои для каждой функции $Q$ ), и таких, что показатели Ляпунова системы $A+Q$, являющиеся функциями $\mu \in M$ и обозначаемые через $\lambda_{1}(\mu ; A+Q) \leqslant \ldots \leqslant \lambda_{n}(\mu ; A+Q)$, не меньше соответствующих показателей Ляпунова системы $A$, т. е. $\lambda_{k}(\mu ; A+Q) \geqslant \lambda_{k}(A), k=\overline{1, n}$, для любого $\mu \in M$. Ставится задача полного описания для каждых $n \in \mathbb{N}$ и метрического пространства $M$ класса пар $(\Lambda(A), \Lambda(\cdot ; A+Q))$, составленных из спектра $\Lambda(A) \in \mathbb{R}^{n}$ системы $A \in \mathcal{M}_{n}$ и из спектра $\Lambda(\cdot ; A+Q): M \rightarrow \mathbb{R}^{n}$ семейства $A+Q$, когда $A$ пробегает множество $\mathcal{M}_{n}$, а матричнозначная функция $Q$ при каждом $A-$ класс $\mathcal{E}_{n}[A](M)$, т. е. класса П $\mathcal{E}_{n}(M)=\left\{(\Lambda(A), \Lambda(\cdot ; A+Q)) \mid A \in \mathcal{M}_{n}, Q \in \mathcal{E}_{n}[A](M)\right\}$. Решение задачи дает следующее утверждение: для любых натурального $n \geqslant 2$ и метрического пространства $M$ пара $(l, F(\cdot))$, где $l=\left(l_{1}, \ldots, l_{n}\right) \in \mathbb{R}^{n}$ и $F(\cdot)=\left(f_{1}(\cdot), \ldots, f_{n}(\cdot)\right): M \rightarrow \mathbb{R}^{n}$, тогда и только тогда принадлежит классу П $\mathcal{E}_{n}(M)$, когда выполняются четыре условия: 1$\left.) l_{1} \leqslant \ldots \leqslant l_{n}, 2\right) f_{1}(\mu) \leqslant \ldots \leqslant f_{n}(\mu)$ для любого $\mu \in M, 3) f_{i}(\mu) \geqslant l_{i}$ для всех $i=\overline{1, n}$ и $\left.\mu \in M, 4\right)$ для любого $i=\overline{1, n}$ функция $f_{i}(\cdot): M \rightarrow \mathbb{R}$ ограничена, и при каждом $r \in \mathbb{R}$ прообраз $f_{i}^{-1}([r,+\infty))$ полуинтервала $[r,+\infty)$ является $G_{\delta}$-множеством. Решение аналогичной задачи описания пар, составленных из индекса es $(A) \in\{0, \ldots, n\}$ экспоненциальной устойчивости системы $A$ и из индекса $\operatorname{es}(\cdot ; A+Q): M \rightarrow\{0, \ldots, n\}$ экспоненциальной устойчивости семейства $A+Q$, т. е. класса $\mathcal{I E}_{n}(M)=\left\{(\operatorname{es}(A), \operatorname{es}(\cdot ; A+Q)) \mid A \in \mathcal{M}_{n}, Q \in \mathcal{E}_{n}[A](M)\right\}$, описывается утверждением: для любых натурального $n \geqslant 2$ и метрического пространства $M$ пара $(d, f(\cdot))$, где $d \in\{0, \ldots, n\}$ и $f: M \rightarrow\{0, \ldots, n\}$, принадлежит классу $\mathcal{I E}_{n}(M)$ тогда и только тогда, когда $f(\mu) \leqslant d$ для любого $\mu \in M$ и при каждом $r \in \mathbb{R}$ прообраз $f^{-1}((-\infty, r])$ полуинтервала $(-\infty, r]$ является $G_{\delta}$-множеством.
\end{abstract}

Ключевые слова: линейная дифференциальная система, показатели Ляпунова, убывающие к нулю возмущения, классы Бэра.

E. A. Barabanov, V. V. Bykov. Description of the linear Perron effect under parametric perturbations exponentially vanishing at infinity.

Let $\mathcal{M}_{n}$ be the set of linear differential systems of order $n \geqslant 2$ whose coefficients are continuous and bounded on the time semiaxis $\mathbb{R}_{+}$. Denote by $\lambda_{1}(A) \leqslant \ldots \leqslant \lambda_{n}(A)$ the Lyapunov exponents of a system $A \in \mathcal{M}_{n}$, by $\Lambda(A)=\left(\lambda_{1}(A), \ldots, \lambda_{n}(A)\right)$ their spectrum, and by es $(A)$ the exponential stability index of $A$ (the dimension of the linear subspace of solutions with negative characteristic exponents). For a system $A \in \mathcal{M}_{n}$ and a metric space $M$, we consider the class $\mathcal{E}_{n}[A](M)$ of continuous $(n \times n)$ matrix-valued functions $Q: \mathbb{R}_{+} \times M \rightarrow \mathbb{R}^{n \times n}$ satisfying the bound $\|Q(t, \mu)\| \leqslant C_{Q} \exp \left(-\sigma_{Q} t\right)$ for all $(t, \mu) \in \mathbb{R}_{+} \times M$, where $C_{Q}$ and $\sigma_{Q}$ are positive constants (possibly different for each function $Q$ ), and such that the Lyapunov exponents of the system $A+Q$, which are functions of $\mu \in M$ and are denoted by $\lambda_{1}(\mu ; A+Q) \leqslant \ldots \leqslant \lambda_{n}(\mu ; A+Q)$, are not less than the corresponding Lyapunov exponents of the system $A$; i.e., $\lambda_{k}(\mu ; A+Q) \geqslant \lambda_{k}(A), k=\overline{1, n}$, for all $\mu \in M$. The problem is to obtain a complete description for each $n \in \mathbb{N}$ and each metric space $M$ of the class of pairs $(\Lambda(A), \Lambda(\cdot ; A+Q))$ composed of the spectrum $\Lambda(A) \in \mathbb{R}^{n}$ of a system $A \in \mathcal{M}_{n}$ and the spectrum $\Lambda(\cdot ; A+Q): M \rightarrow \mathbb{R}^{n}$ of a family $A+Q$, where $A$ ranges over $\mathcal{M}_{n}$ and the matrix-valued function $Q$ ranges over the class $\mathcal{E}_{n}[A](M)$ for each $A$, i.e., of the class $\Pi \mathcal{E}_{n}(M)=\left\{(\Lambda(A), \Lambda(\cdot ; A+Q)) \mid A \in \mathcal{M}_{n}, Q \in \mathcal{E}_{n}[A](M)\right\}$. The solution of this problem is provided by the following statement: for each integer $n \geqslant 2$ and every metric space $M$, a pair $(l, F(\cdot))$, where $l=\left(l_{1}, \ldots, l_{n}\right) \in \mathbb{R}^{n}$ and $F(\cdot)=\left(f_{1}(\cdot), \ldots, f_{n}(\cdot)\right): M \rightarrow \mathbb{R}^{n}$, belongs to the class $\Pi \mathcal{E}_{n}(M)$ if and only if the following conditions are met: (1) $l_{1} \leqslant \ldots \leqslant l_{n},(2) f_{1}(\mu) \leqslant \ldots \leqslant f_{n}(\mu)$ for all $\mu \in M$, (3) $f_{i}(\mu) \geqslant l_{i}$ for all $i=\overline{1, n}$ and $\mu \in M$, (4) for each $i=\overline{1, n}$, the function $f_{i}(\cdot): M \rightarrow \mathbb{R}$ is bounded and, for any $r \in \mathbb{R}$, the preimage $f_{i}^{-1}([r,+\infty))$ of the half-interval $[r,+\infty)$ is a $G_{\delta}$-set. The solution of the similar problem of describing the pairs composed of the exponential stability index es $(A) \in\{0, \ldots, n\}$ of a system $A$ and the exponential stability index es $(\cdot ; A+Q): M \rightarrow\{0, \ldots, n\}$ of a family $A+Q$, i.e., the class $\mathcal{I E}_{n}(M)=\left\{(\operatorname{es}(A), \mathrm{es}(\cdot ; A+Q)) \mid A \in \mathcal{M}_{n}, Q \in \mathcal{E}_{n}[A](M)\right\}$, is contained in the following statement: for any positive integer $n \geqslant 2$ and every metric space $M$, a pair $(d, f(\cdot))$, where $d \in\{0, \ldots, n\}$ and $f: M \rightarrow\{0, \ldots, n\}$, belongs to the class $\mathcal{I E}_{n}(M)$ if and only if $f(\mu) \leqslant d$ for all $\mu \in M$ and, for any $r \in \mathbb{R}$, the preimage $f^{-1}((-\infty, r])$ of the half-interval $(-\infty, r]$ is a $G_{\delta}$-set. 
Keywords: linear differential system, Lyapunov exponents, perturbations vanishing at infinity, Baire classes.

MSC: 34D08, 34D10

DOI: $10.21538 / 0134-4889-2019-25-4-31-43$

\section{Введение}

Для заданного натурального $n$ через $\mathcal{M}_{n}$ обозначим множество линейных дифференциальных систем

$$
\dot{x}=A(t) x, \quad x \in \mathbb{R}^{n}, \quad t \in \mathbb{R}_{+} \equiv[0,+\infty),
$$

с ограниченными и непрерывными на временно́й полуоси $\mathbb{R}_{+}$коэффициентами. Обозначим через $\lambda_{1}(A) \leqslant \ldots \leqslant \lambda_{n}(A)$ показатели Ляпунова [1, с. 27; 2, гл. III, §4] системы (1), через $\Lambda(A)=\left(\lambda_{1}(A), \ldots, \lambda_{n}(A)\right)$ - ее спектр, а через es $(A)$ - индекс экспоненциальной устойчивости, т. е. размерность линейного пространства решений этой системы, имеющих отрицательные характеристические показатели. Далее мы отождествляем систему (1) с матричнозначной функцией $A(\cdot)$ и пишем $A \in \mathcal{M}_{n}$.

В работе [3] О. Перрон построил пример системы $A \in \mathcal{M}_{2}$ с отрицательными показателями Ляпунова, для которой существует такая непрерывная на полуоси $(2 \times 2)$-матрица $Q(t)$, экспоненциально убывающая к нулю на бесконечности, что старший показатель Ляпунова $\lambda_{2}(A+Q)$ возмущенной системы $(A+Q) \in \mathcal{M}_{2}$ положителен (в частности, $\lambda_{2}(A+Q)>\lambda_{2}(A)$ ), а младший $\lambda_{1}(A+Q)$ совпадает с показателем $\lambda_{1}(A)$ невозмущенной системы. Таким образом, в примере Перрона индекс экспоненциальной устойчивости исходной системы равен двум, а возмущенной - единице, т. е. имеет место потеря устойчивости. О. Перроном [4] построен также пример системы $A \in \mathcal{M}_{2}$ с отрицательными показателями Ляпунова и такого ее определенного в произведении $\mathbb{R}_{+} \times G\left(G\right.$ - окрестность нуля в $\left.\mathbb{R}^{2}\right)$ непрерывного по совокупности переменных возмущения $f(t, x)$ высшего порядка малости (т. е. $\|f(t, x)\| \leqslant$ const $\|x\|^{m}, m=$ const $>1$ ), что показатель Ляпунова любого имеющего в начальный момент $t=0$ ненулевую первую компоненту решения возмущенной системы $\dot{x}=A(t) x+f(t, x)$ больше некоторого положительного числа, а показатель Ляпунова решений с нулевой в начальный момент первой компонентой тот же, что и у невозмущенной линейной системы.

Эти примеры Перрона послужили отправной точкой многочисленных исследований влияния различных классов линейных и нелинейных возмущений на показатели Ляпунова систем из $\mathcal{M}_{n}$, а результаты, полученные в этом направлении, составляют существенную часть современной теории показателей Ляпунова. Эффект изменения значений показателей Ляпунова системы из $\mathcal{M}_{n}$ при тех или иных "малых" ее возмущениях назван в монографии [5, гл. 4] эффектом Перрона. Позднее, начиная с работы [6], это название - эффект Перрона - было усвоено только той ситуации (ее, формально говоря, и рассмотрел О. Перрон), при которой возмущения не уменьшают показатели Ляпунова исходной системы (этой терминологии мы и следуем в дальнейшем). В отличие от работ [5;6], в которых эффект Перрона, как в [4], рассматривался при возмущениях высшего порядка малости, мы в соответствии с работой [3] рассматриваем линейные убывающие (в частности, экспоненциально) к нулю возмущения матриц коэффициентов систем из $\mathcal{M}_{n}$ и в этом случае называем эффект Перрона линейным.

Для дальнейшего важно отметить, что построенная в работе [3] матрица-возмущение имела вид $\mu Q(t)$, где $\mu$ - вещественный параметр; доказано, что для любого $\mu \neq 0$ при таком возмущении старший показатель Ляпунова возмущенной системы положителен, а младший не изменяется. Имея в виду это обстоятельство, далее, зафиксировав некоторое метрическое пространство $M$, будем рассматривать семейства линейных дифференциальных систем вида

$$
\dot{x}=(A(t)+Q(t, \mu)) x, \quad x \in \mathbb{R}^{n}, \quad t \in \mathbb{R}_{+},
$$

где $A \in \mathcal{M}_{n}$, а $Q(\cdot, \cdot): \mathbb{R}_{+} \times M \rightarrow \mathbb{R}^{n \times n}-$ непрерывная по совокупности переменных матричнозначная функция. При каждом фиксированном в семействе (2) значении параметра $\mu \in M$ 
получаем линейную дифференциальную систему с ограниченными (своей, вообще говоря, для каждого $\mu$ постоянной) и непрерывными на полуоси коэффициентами, показатели Ляпунова которой обозначим через $\lambda_{1}(\mu ; A+Q) \leqslant \ldots \leqslant \lambda_{n}(\mu ; A+Q)$. Таким образом, показатели Ляпунова семейства (2) - функции параметра $\mu \in M$; в частности, определен спектр семейства (2) вектор-функция $\Lambda(\cdot ; A+Q) \equiv\left(\lambda_{1}(\cdot ; A+Q), \ldots, \lambda_{n}(\cdot ; A+Q)\right): M \rightarrow \mathbb{R}^{n}$.

\section{1. Постановка задачи. Основной результат}

Через $\mathcal{E}_{n}(M)$ обозначим класс непрерывных по совокупности переменных матричнозначных функций $Q(\cdot, \cdot): \mathbb{R}_{+} \times M \rightarrow \mathbb{R}^{n \times n}$, экспоненциально убывающих к нулю при $t \rightarrow+\infty$ равномерно относительно $\mu \in M$, т. е.

$$
\varlimsup_{t \rightarrow+\infty} \ln \sup _{\mu \in M}\|Q(t, \mu)\|^{1 / t}<0 .
$$

Для каждой системы $A \in \mathcal{M}_{n}$ через $\mathcal{E}_{n}[A](M)$ обозначим класс тех возмущений $Q \in \mathcal{E}_{n}(M)$, которые не уменьшают ее показатели Ляпунова, т. е. для любых системы $A \in \mathcal{M}_{n}$ и ее возмущения $Q \in \mathcal{E}_{n}[A](M)$ выполняется неравенство $\inf _{\mu \in M} \lambda_{i}(\mu ; A+Q) \geqslant \lambda_{i}(A)$ при всех $i=\overline{1, n}$. Очевидно, что для любой системы $A \in \mathcal{M}_{n}$ класс $\mathcal{E}_{n}[A](M)$ не пуст, поскольку ему принадлежит тождественно нулевая матрица.

Ставится задача полного дескриптивно-множественного описания для каждых $n \in \mathbb{N}$ и метрического пространства $M$ класса пар $(\Lambda(A), \Lambda(\cdot ; A+Q))$, составленных из спектра $\Lambda(A)$ системы $A \in \mathcal{M}_{n}$ и вектор-функции $\Lambda(\cdot ; A+Q)$, когда $A$ пробегает множество $\mathcal{M}_{n}$, а матричнозначная функция $Q$ при каждом $A-$ класс $\mathcal{E}_{n}[A](M)$, т. е. класса

$$
\Pi \mathcal{E}_{n}(M)=\left\{(\Lambda(A), \Lambda(\cdot ; A+Q)) \mid A \in \mathcal{M}_{n}, Q \in \mathcal{E}_{n}[A](M)\right\} .
$$

Отметим, что полное описание класса

$$
\Lambda \mathcal{E}_{n}(M)=\left\{\Lambda(\cdot ; A+Q) \mid A \in \mathcal{M}_{n}, Q \in \mathcal{E}_{n}[A](M)\right\},
$$

составленного из вторых элементов пар класса П $\mathcal{E}_{n}(M)$, фактически получено в работе [7].

Очевидно, что решение поставленной задачи будет содержать как частный случай пример Перрона и будет описывать с дескриптивно-множественной точки зрения в том числе и все возможные ситуации, при которых экспоненциально устойчивая линейная система под действием параметрических экспоненциально убывающих возмущений становится неустойчивой. Например, как следует из теоремы работы, существуют такая система $A \in \mathcal{M}_{2}$ со старшим показателем Ляпунова $\lambda_{2}(A)$, равным -1 , и такое ее возмущение $Q \in \mathcal{E}_{2}[A](\mathbb{R})$, что старший показатель $\lambda_{2}(A+Q)$ Ляпунова возмущенной системы при $\mu$ рациональном равен -1 , а при $\mu$ иррациональном равен 1.

Отметим, что направление в теории показателей Ляпунова, ставящее своей целью изучение зависимости от параметра асимптотических свойств и характеристик параметрических дифференциальных систем, возникло благодаря В. М. Миллионщикову, начавшему систематические исследования в этом направлении серией работ, из которых укажем только работу [8]. Ему же мы обязаны пониманием того, что адекватным языком описания такой зависимости является язык теории Бэра разрывных функций [9]. Подчеркнем, что речь идет о полном описании всех возможных типов поведения тех или иных свойств или характеристик при изменении параметров системы, а не об установлении достаточных условий того или иного характера их поведения. $\mathrm{K}$ настоящему времени в этом направлении получено большое количество результатов.

Так как при $n=1$ для любого метрического пространства $M$ и любого семейства $Q \in$

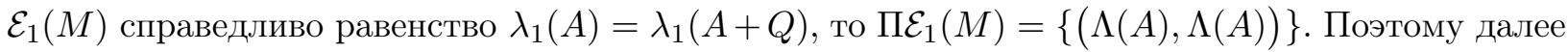
считаем, что $n>1$. 
Напомним, что функция $f: M \rightarrow \mathbb{R}$ называется [10, с. 224] функцией класса $\left({ }^{*}, G_{\delta}\right)$, если для любого $r \in \mathbb{R}$ прообраз $f^{-1}([r,+\infty))$ полуинтервала $[r,+\infty)$ является $G_{\delta}$-множеством метрического пространства $M$. В частности, класс $\left({ }^{*}, G_{\delta}\right)$ - подкласс второго класса Бэра $[10$, c. 248].

Полное описание класса $\Pi \mathcal{E}_{n}(M)$ для любых $n \in \mathbb{N}, n>1$, и пространства $M$ дает следующее утверждение, которое было анонсировано в [11].

Теорема. Пусть $n$ - натуральное число, большее единицы, и $M$ - метрическое пространство. Пара $(l, F(\cdot))$, где $l=\left(l_{1}, \ldots, l_{n}\right) \in \mathbb{R}^{n}$ u $F(\cdot)=\left(f_{1}(\cdot), \ldots, f_{n}(\cdot)\right): M \rightarrow \mathbb{R}^{n}$, тогда и только тогда принадлежит классу П $\mathcal{E}_{n}(M)$, когда выполняются следующие условия:

1) $l_{1} \leqslant \ldots \leqslant l_{n}$

2) $f_{1}(\mu) \leqslant \ldots \leqslant f_{n}(\mu)$ для любого $\mu \in M$;

3) $f_{i}(\mu) \geqslant l_{i}$ для всех $\mu \in M$ u $i=\overline{1, n}$;

4) для любого $i=\overline{1, n}$ функиия $f_{i}(\cdot): M \rightarrow \mathbb{R}$ ограничена и принадлежит классу $\left({ }^{*}, G_{\delta}\right)$.

Следствие 1. Пусть $n$ - натуральное число, большее единицы, и $M$-отрезок вещественной прямой. Тогда для всякой пары $(l, F(\cdot)) \in \Pi \mathcal{E}_{n}(M)$ существуют система $A \in \mathcal{M}_{n}$ и аналитическое по параметру $\mu \in M$ ее возмущение $Q \in \mathcal{E}_{n}[A](M)$ такие, что $\Lambda(A)=l$ u $\Lambda(\cdot ; A+Q)=F$.

Поставив каждому $\mu \in M$ в соответствие индекс экспоненциальной устойчивости системы (2), получим функцию еs( $\cdot ; A): M \rightarrow \mathcal{Z}_{n}$, где $\mathcal{Z}_{n} \equiv\{0, \ldots, n\}$. Естественно возникает задача описания класса пар, составленных из индексов экспоненциальной устойчивости исходной и возмущенной систем, т. е. класса $\mathcal{I E}_{n}(M)=\left\{(\operatorname{es}(A), \operatorname{es}(\cdot ; A+Q)) \mid A \in \mathcal{M}_{n}, Q \in \mathcal{E}_{n}[A](M)\right\}$. Описание этого класса содержит следующее

Следствие 2. Пусть $n$ - натуральное число, большее единицы, а $M$ - метрическое пространство. Пара $(d, f(\cdot))$, где $d \in \mathcal{Z}_{n}$ u $f: M \rightarrow \mathcal{Z}_{n}$ принадлежит классу $\mathcal{I E}_{n}(M)$ тогда и только тогда, когда выполняются условия: а) $f(\mu) \leqslant d$ для любого $\mu \in M$, б) функиия (-f) принадлежит классу $\left({ }^{*}, G_{\delta}\right)$.

\section{2. Доказательство основных результатов}

Сначала сформулируем и докажем три вспомогательных утверждения. Наделим пространство $\mathbb{R}^{n}$ наиболее удобной для дальнейшего нормой $\|x\|=\max _{1 \leq i \leq n}\left|x_{i}\right|, x=\left(x_{1}, \ldots, x_{n}\right) \in \mathbb{R}^{n}$, а пространство $n \times n$ матриц - соответствующей операторной нормой

$$
\|Y\|=\sup _{\|x\|=1}\|Y x\|, \quad Y \in \mathbb{R}^{n \times n} .
$$

Лемма 1. Пусть заданы числа $a_{1}, \ldots, a_{n} \in \mathbb{R}$ и отрезок $[c, d] \subset(0,+\infty)$. Тогда для любых $i \in\{1, \ldots, n\}$ и решения $x(\cdot)=\left(x_{1}(\cdot), \ldots, x_{n}(\cdot)\right)^{\mathrm{T}}$ системы

$$
\dot{x}=\operatorname{diag}\left[a_{1}, \ldots, a_{n}\right] x, \quad x \in \mathbb{R}^{n}, \quad t \in[c, d],
$$

функиия $\chi_{i}^{x}:[c, d] \rightarrow \mathbb{R} \sqcup\{-\infty\}$, определяемая равенством $\chi_{i}^{x}(t)=\ln \left|x_{i}(t)\right|^{1 / t}, t \in[c, d]$, монотонна (вообще говоря, нестрого).

Д о к а з а т е л ь с т в о. Для любых $i \in\{1, \ldots, n\}$ и решения $x(\cdot)$ рассматриваемой системы справедливо равенство $x_{i}(t)=e^{a_{i}(t-c)} x_{i}(c), t \in[c, d]$. Следовательно, если $x_{i}(c) \neq 0$, то $\chi_{i}^{x}(t)=a_{i}+\left(\ln \left|x_{i}(c)\right|-a_{i} c\right) / t, t \in[c, d]$, а если $x_{i}(c)=0$, то $\chi_{i}^{x}(t)=-\infty$ при всех $t \in[c, d]$.

Лемма доказана.

О п р е д е л е н и е. Пусть $\mathrm{S} \subset \mathbb{R}_{+}$- неограниченное множество. Характеристическим показателем вектор-функции $f: \mathrm{S} \rightarrow \mathbb{R}^{n}$ называется величина (считаем $\ln 0=-\infty$ )

$$
\lambda[f]=\varlimsup_{\mathrm{S} \ni t \rightarrow+\infty} \ln \|f(t)\|^{1 / t} .
$$


Лемма 2. Пусть $\mathrm{S} \subset \mathbb{R}_{+}$- неограниченное множество. Тогда для любой вектор-функиии $x(\cdot)=\left(x_{1}(\cdot), \ldots, x_{n}(\cdot)\right)^{\mathrm{T}}: \mathrm{S} \rightarrow \mathbb{R}^{n}$ справедливо равенство

$$
\lambda[x]=\max _{1 \leq i \leq n} \lambda\left[x_{i}\right] .
$$

Д о к а з а т е л ь с т в о. По определению

$$
\lambda[x]=\varlimsup_{\mathrm{S} \ni t \rightarrow+\infty} \ln \left(\max _{1 \leq i \leq n}\left|x_{i}(t)\right|\right)^{1 / t} .
$$

Для любых $i \in\{1, \ldots, n\}$ и $t>0$ из неравенства $\max _{1 \leq l \leq n}\left|x_{l}(t)\right| \geq\left|x_{i}(t)\right|$ вытекает неравенство $\ln \left(\max _{1 \leq l \leq n}\left|x_{l}(t)\right|\right)^{1 / t} \geq \ln \left|x_{i}(t)\right|^{1 / t}$, откуда, переходя к верхнему пределу при $t \rightarrow+\infty$ по множеству $\mathrm{S}$, получаем неравенство $\lambda[x] \geq \lambda\left[x_{i}\right]$. Следовательно, $\lambda[x] \geq \max _{1 \leq i \leq n} \lambda\left[x_{i}\right]$.

Установим обратное неравенство. Пусть, напротив, для некоторого числа $r \in \mathbb{R}$ справедливо соотношение $\lambda[x]>r>\max _{1 \leq i \leq n} \lambda\left[x_{i}\right]$. Значит, для каждого $i \in\{1, \ldots, n\}$ существует такое $T_{i}>0$, что при всех $t \geq T_{i}$ выполнено неравенство $\ln \left|x_{i}(t)\right|^{1 / t}<r$. Положим $T=\max _{1 \leq i \leq n} T_{i}$. Тогда при всех $t \geq T$ справедливо неравенство $\ln \|x(t)\|^{1 / t}<r$. Переходя в последнем неравенстве к верхнему пределу при $t \rightarrow+\infty$ по множеству $\mathrm{S}$, получим, что $\lambda[x] \leq r$. Пришли к противоречию.

Лемма доказана.

Лемма 3. Пусть $A \in \mathcal{M}_{n}$, а матричнозначная функиия $Q(\cdot, \cdot): \mathbb{R}_{+} \times M \rightarrow \mathbb{R}^{n \times n}$ непрерывна и при каждом $\mu \in M$ удовлетворяет условию $\lim _{t \rightarrow+\infty}\|Q(t, \mu)\|=0$. Тогда каждая из функиий $\lambda_{i}(\cdot ; A+Q), i=\overline{1, n}$, ограничена и принадлежит классу $\left({ }^{*}, G_{\delta}\right)$.

Д о к а з а т е л ь с т в о. Зафиксируем произвольное $i \in\{1, \ldots, n\}$. При каждом $\mu \in M$ из равенства $\lim _{t \rightarrow+\infty}\|Q(t, \mu)\|=0$ вытекает, что существует такое $T>0$, что для всех $t \geq T$ выполнено неравенство $\|Q(t, \mu)\| \leq 1$. В силу известной оценки для показателей Ляпунова $[2$, гл. III, §3] справедливо включение $\lambda_{i}(A+Q) \in[-(a+1), a+1]$, где $a=\sup \left\{\|A(t)\|: t \in \mathbb{R}_{+}\right\}$. Следовательно, функция $\lambda_{i}(\cdot ; A+Q)$ ограничена. Принадлежность этой функции классу $\left({ }^{*}, G_{\delta}\right)$ по существу вытекает из [12, леммы 6-9] (см. также доказательство теоремы [13]).

Лемма доказана.

Д о к а з а т е л ь с т в о теоремы разобьем на шаги.

1. Установим сначала необходимость условий теоремы. Условия 1)-3) вытекают непосредственно из определений, а условие 4) - из леммы 3.

2. Докажем достаточность. Пусть $l \in \mathbb{R}^{n}$ и $F=\left(f_{1}(\cdot), \ldots, f_{n}(\cdot)\right)$ удовлетворяют условиям 1)-4). В силу результата [14] (см. также [15, замечание 2]) для каждого $i \in\{1, \ldots, n\}$ существует последовательность непрерывных функций $f_{m}^{i}: M \rightarrow \mathbb{R}, m \in \mathbb{N}$, такая, что справедливо представление

$$
f_{i}(\mu)=\varlimsup_{m \rightarrow \infty} f_{m}^{i}(\mu), \quad \mu \in M
$$

Положим $C=\sup \{\|F(\mu)\|: \mu \in M\}+1$. Без ограничения общности можно считать, что для всех $i=\overline{1, n}, m \in \mathbb{N}$ и $\mu \in M$ выполнено неравенство $f_{m}^{i}(\mu) \leq C-1$ (в противном случае заменим функцию $f_{m}^{i}$ функцией $\left.\min \left\{f_{m}^{i}(\cdot), C-1\right\}\right)$.

Для каждого $m \in \mathbb{N}_{0}$ определим функцию $\sigma_{m}: M \rightarrow \mathbb{R}$ равенством

$$
\sigma_{m}(\mu)=\left(6 C+4 l_{i}-6 f_{\max \{q, 1\}}^{i}(\mu)\right) / 5, \quad \mu \in M,
$$

где $q \in \mathbb{Z}$ и $i \in\{1, \ldots, n\}$ однозначно определяются из условия $m=q n+i$.

3. Зададим последовательность $T_{m}^{j}, m \in \mathbb{N}_{0}, j=\overline{0,6}$, неотрицательных целых чисел рекуррентно равенствами $T_{0}^{0}=0$ и

$$
T_{m+1}^{0}=T_{m}^{6}, \quad T_{m}^{j}=T_{m}^{j-1}+\left\{\begin{array}{ccl}
1, & \text { если } & j=2,5, \\
2^{m+1}, & \text { если } & j=1,3,4,6,
\end{array} \quad m \in \mathbb{N}_{0}, \quad j=\overline{1,6} .\right.
$$


Легко видеть, что $T_{m}^{0}=4\left(2^{m+1}-2\right)+2 m, m \in \mathbb{N}_{0}$. Для упрощения записи через $\Delta_{m}^{j}, j=\overline{1,6}$, обозначим полуинтервал $\left[T_{m}^{j-1}, T_{m}^{j}\right)$, через $\Delta_{m}-$ полуинтервал $\left[T_{m}^{0}, T_{m+1}^{0}\right)$, а через $\bar{\Delta}_{m}^{j}$ и $\bar{\Delta}_{m}-$ соответствующие отрезки.

Для всякой тройки чисел $\alpha=(a, b, c) \in \mathbb{R}^{3}$ определим на отрезке $\bar{\Delta}_{m}$ матричнозначную функцию $A[\alpha ; m]$ при помощи равенства

$$
A[\alpha ; m](t)= \begin{cases}\operatorname{diag}[0,-c] & \text { при } t \in \Delta_{m}^{1} \sqcup \Delta_{m}^{4}, \\ O_{2} & \text { при } t \in \Delta_{m}^{2} \sqcup \Delta_{m}^{5}, \\ \operatorname{diag}[0, c] & \text { при } t \in \Delta_{m}^{3}, \\ \operatorname{diag}[a, b+c] & \text { при } t \in \bar{\Delta}_{m}^{6},\end{cases}
$$

а для всякого $\sigma>0$ - матричнозначную функцию $Q[\sigma ; m]$ при помощи равенства

$$
Q[\sigma ; m](t)= \begin{cases}\left(\begin{array}{cc}
0 & 0 \\
(-1)^{j} e^{-\sigma T_{m}^{2}} & 0
\end{array}\right) & \text { при } t \in \Delta_{m}^{j}, \quad j \in\{2,5\}, \\
O_{2} & \text { при остальных } t \in \bar{\Delta}_{m},\end{cases}
$$

где $O_{2}-$ нулевая $(2 \times 2)$-матрица. Положим $C[\alpha, \sigma ; m]=A[\alpha ; m]+Q[\sigma ; m]$. Тогда для матрицы Коши $X_{C[\alpha, \sigma ; m]}(\cdot, \cdot)$ системы

$$
\dot{x}=C[\alpha, \sigma ; m](t) x, \quad x=\left(x_{1}, x_{2}\right)^{\mathrm{T}} \in \mathbb{R}^{2}, \quad t \in \bar{\Delta}_{m},
$$

непосредственными вычислениями получаем соотношения

$$
\begin{gathered}
X_{C[\alpha, \sigma ; m]}\left(T_{m}^{1}, T_{m}^{0}\right)=X_{C[\alpha, \sigma ; m]}\left(T_{m}^{5}, T_{m}^{0}\right)=\left(\begin{array}{cc}
1 & 0 \\
0 & e^{-c 2^{m+1}}
\end{array}\right), \\
X_{C[\alpha, \sigma ; m]}\left(T_{m}^{2}, T_{m}^{0}\right)=X_{C[\alpha, \sigma ; m]}\left(T_{m}^{4}, T_{m}^{0}\right)=\left(\begin{array}{cc}
1 & 0 \\
e^{-\sigma T_{m}^{2}} & e^{-c 2^{m+1}}
\end{array}\right), \\
X_{C[\alpha, \sigma ; m]}\left(T_{m}^{3}, T_{m}^{0}\right)=\left(\begin{array}{cc}
1 & 0 \\
e^{c 2^{m+1}-\sigma T_{m}^{2}} & 1
\end{array}\right), \quad X_{C[\alpha, \sigma ; m]}\left(T_{m}^{6}, T_{m}^{0}\right)=\left(\begin{array}{cc}
e^{a 2^{m+1}} & 0 \\
0 & e^{b 2^{m+1}}
\end{array}\right) .
\end{gathered}
$$

4. Построим кусочно-постоянную матричнозначную функцию $\widetilde{A}(\cdot)$ и семейство кусочнопостоянных матричнозначных функщий $\widetilde{Q}(\cdot, \mu), \mu \in M$, со следующими свойствами:

1) все точки разрыва функций $\widetilde{A}(\cdot)$ и $\widetilde{Q}(\cdot, \mu), \mu \in M$, содержатся в множестве $\left\{T_{m}^{j}: m \in\right.$ $\left.\mathbb{N}_{0}, j=\overline{1,6}\right\}$

2) функция $\widetilde{Q}(\cdot, \mu)$ экспоненциально стремится к нулю при $t \rightarrow+\infty$ равномерно относительно $\mu \in M$;

3) спектр $\Lambda(\widetilde{A})$ показателей Ляпунова системы $\widetilde{A}$ совпадает с вектором $l$;

4) спектр $\Lambda(\cdot ; \widetilde{A}+\widetilde{Q})$ показателей Ляпунова семейства $\widetilde{A}+\widetilde{Q}$ совпадает с вектор-функцией $F(\cdot)$.

4.1. Рассмотрим сначала случай $l_{1}=0$. Для каждого $m \in \mathbb{N}_{0}$ определим на полуинтервале $\Delta_{m}$ систему $\widetilde{A}(\cdot)$ равенствами

$$
\begin{aligned}
& \dot{x}_{j}=l_{j} x_{j}, \quad \text { если } \quad j \notin\{\theta(m), \theta(m+1)\}, \\
& \dot{y}=A\left[a_{m}, b_{m}, 6 C ; m\right](t) y,
\end{aligned}
$$

а систему $\widetilde{Q}(\cdot, \mu)$ при всяком $\mu \in M-$ равенствами (6) и

$$
\dot{y}=Q\left[\sigma_{m}(\mu) ; m\right](t) y .
$$

В равенствах (6)-(8) обозначены $y=\left(x_{\theta(m)}, x_{\theta(m+1)}\right)^{\mathrm{T}}, a_{m}=\left(4+2^{-m}\right) l_{\theta(m)}, b_{m}=\left(4+2^{-m}\right) l_{\theta(m+1)}$ и $\theta: \mathbb{N}_{0} \rightarrow\{1, \ldots, n\}-$ функция, которая задается условием: $\theta(m) \equiv m(\bmod n)$. 
Свойство 1) выполнено по построению. Для доказательства свойства 2) заметим, что при всех $m \in \mathbb{N}_{0}$ и $\mu \in M$ выполнено неравенство $\sigma_{m}(\mu) \geq 6 / 5$. Следовательно,

$$
\varlimsup_{t \rightarrow+\infty} \ln \sup _{\mu \in M}\|\widetilde{Q}(t, \mu)\|^{1 / t} \leq \lim _{m \rightarrow+\infty}-6 T_{m}^{2} /\left(5 T_{m}^{5}\right)=-6 / 7 .
$$

4.2. Зафиксируем $\mu \in M$ и вычислим показатели Ляпунова системы $\widetilde{C}(\cdot, \mu) \equiv \widetilde{A}(\cdot)+\widetilde{Q}(\cdot, \mu)$. Рассмотрим произвольное ненулевое решение $x(\cdot)$ этой системы. Поскольку $\|\widetilde{C}(t, \mu)\| \leq 1$ при всех $t \in \bar{\Delta}_{m}^{j}, j \in\{2,5\}$, из равенства $x(t)=X_{\widetilde{C}(\cdot, \mu)}\left(t, T_{m}^{j}\right) x\left(T_{m}^{j}\right)$ в силу известной оценки для нормы матрицы Коши [16, (3.3)] получаем двойное неравенство

$$
\ln \left\|x\left(T_{m}^{j}\right)\right\|-1 \leq \ln \|x(t)\| \leq \ln \left\|x\left(T_{m}^{j}\right)\right\|+1, \quad t \in \bar{\Delta}_{m}^{j}, \quad m \in \mathbb{N}_{0}, \quad j \in\{2,5\} .
$$

Следовательно, характеристический показатель решения $x(\cdot)$ совпадает с характеристическим показателем $\lambda\left[\left.x\right|_{\mathrm{T}}\right]$ его сужения на множество $\mathrm{T}=\bigcup\left\{\bar{\Delta}_{m}^{j}: m \in \mathbb{N}_{0}, j \neq 2,5\right\}$. Из леммы 2 следует, что упомянутый показатель равен наибольшему из характеристических показателей $\lambda\left[\left.x_{i}\right|_{\mathrm{T}}\right], i=\overline{1, n}$. Так как на отрезках $\bar{\Delta}_{m}^{j}, m \in \mathbb{N}_{0}, j \in\{1,3,4,6\}$, составляющих множество Т, система $\widetilde{C}(\cdot, \mu)$ является автономной и диагональной, то в силу леммы 1 каждая из функций $\chi_{i}^{x}, i=\overline{1, n}$, монотонна, поэтому верхний предел в определении характеристического показателя $\lambda\left[\left.x_{i}\right|_{\mathrm{T}}\right]$ можно вычислять по последовательности концов отрезков $\bar{\Delta}_{m}^{j}, m \in \mathbb{N}_{0}, j=\overline{1,6}$ :

$$
\lambda\left[\left.x_{i}\right|_{\mathrm{T}}\right]=\varlimsup_{q \rightarrow \infty} \ln \left|x_{i}\left(T_{q}\right)\right|^{1 / t}, \text { где } T_{6 m+j}=T_{m}^{j}, \quad m \in \mathbb{N}_{0}, \quad j=\overline{1,6} .
$$

Учитывая сказанное и применяя лемму 2 к сужению функции $x(\cdot)$ на множество $\left\{T_{q}: q \in \mathbb{N}\right\}$, получаем цепочку равенств

$$
\lambda[x]=\lambda\left[\left.x\right|_{\mathrm{T}}\right]=\max _{1 \leq i \leq n} \lambda\left[\left.x_{i}\right|_{\mathrm{T}}\right]=\max _{1 \leq i \leq n} \varlimsup_{q \rightarrow \infty} \ln \left|x_{i}\left(T_{q}\right)\right|^{1 / t}=\varlimsup_{q \rightarrow \infty} \frac{1}{t} \ln \left\|x\left(T_{q}\right)\right\| .
$$

4.3. Зафиксируем произвольное $i \in\{1, \ldots, n\}$. Через $e^{i}$ обозначим $i$-й единичный вектор пространства $\mathbb{R}^{n}$. Вычислим характеристический показатель $\lambda\left[x^{i}\right]$ решения $x^{i}(\cdot)$ рассматриваемой системы, выходящего в момент времени $t=0$ из вектора $e^{i}$.

Заметим, что из последнего равенства в (5) и задания систем (6)-(8) вытекает, что для $i$-й компоненты любого решения $x(\cdot)$ рассматриваемой системы при всех $m \in \mathbb{N}_{0}$ выполнено соотношение $x_{i}\left(T_{m+1}^{0}\right)=x_{i}\left(T_{m}^{0}\right) \exp \left(l_{i}\left(T_{m+1}^{0}-T_{m}^{0}\right)\right)$. Следовательно, справедливо равенство

$$
x_{i}\left(T_{m}^{0}\right)=\exp \left(l_{i} T_{m}^{0}\right) x_{i}\left(T_{0}^{0}\right), \quad m \in \mathbb{N}_{0},
$$

из которого и определения систем (6)-(8) следует, что если ни одно из чисел $m-i$ и $m-i+1$ не делится на $n$, то функция $\chi_{i}(t) \equiv t^{-1} \ln \left\|x^{i}(t)\right\|, t>0$, на отрезке $\bar{\Delta}_{m}$ тождественно равна $l_{i}$. Следовательно, при вычислении верхнего предела в определении величины $\lambda\left[x^{i}\right]$ можно ограничиться концами только тех отрезков $\bar{\Delta}_{m}^{j}, j=\overline{1,6}$, для которых одно из чисел $m-i$ или $m-i+1$ делится на $n$.

Положим $m_{q}^{i}=q n+i, q \in \mathbb{N}_{0}, i=\overline{1, n}$. Из (5)-(8) получаем соотношения

$$
\begin{gathered}
\left\|x^{i}\left(T_{m_{q}^{i}}^{5}\right)\right\|=\left\|x^{i}\left(T_{m_{q}^{i}}^{4}\right)\right\|=\left\|x^{i}\left(T_{m_{q}^{i}}^{2}\right)\right\|=\left\|x^{i}\left(T_{m_{q}^{i}}^{1}\right)\right\|=\left\|x^{i}\left(T_{m_{q}^{i}}^{0}\right)\right\|, \\
\left\|x^{i}\left(T_{m_{q}^{i}-1}^{5}\right)\right\|=\left\|x^{i}\left(T_{m_{q}^{i}-1}^{4}\right)\right\|=\left\|x^{i}\left(T_{m_{q}^{i}-1}^{2}\right)\right\|=\left\|x^{i}\left(T_{m_{q}^{i}-1}^{1}\right)\right\| \leq\left\|x^{i}\left(T_{m_{q}^{i}-1}^{3}\right)\right\|=\left\|x^{i}\left(T_{m_{q}^{i}-1}^{0}\right)\right\|,
\end{gathered}
$$

откуда с учетом (10) вытекают цепочки неравенств

$$
\begin{gathered}
\varlimsup_{q \rightarrow \infty} \chi_{i}\left(T_{m_{q}^{i}}^{5}\right) \leq \varlimsup_{q \rightarrow \infty} \chi_{i}\left(T_{m_{q}^{i}}^{4}\right) \leq \varlimsup_{q \rightarrow \infty} \chi_{i}\left(T_{m_{q}^{i}}^{2}\right) \leq \varlimsup_{q \rightarrow \infty} \chi_{i}\left(T_{m_{q}^{i}}^{1}\right) \leq \varlimsup_{q \rightarrow \infty} \chi_{i}\left(T_{m_{q}^{i}}^{0}\right)=l_{i}, \\
\varlimsup_{q \rightarrow \infty} \chi_{i}\left(T_{m_{q}^{i}-1}^{5}\right) \leq \varlimsup_{q \rightarrow \infty} \chi_{i}\left(T_{m_{q}^{i}-1}^{4}\right) \leq \varlimsup_{q \rightarrow \infty} \chi_{i}\left(T_{m_{q}^{i}-1}^{2}\right) \leq \varlimsup_{q \rightarrow \infty} \chi_{i}\left(T_{m_{q}^{i}-1}^{1}\right)
\end{gathered}
$$




$$
\leq \varlimsup_{q \rightarrow \infty} \chi_{i}\left(T_{m_{q}^{i}-1}^{3}\right) \leq \varlimsup_{q \rightarrow \infty} \chi_{i}\left(T_{m_{q}^{i}-1}^{0}\right)=\varlimsup_{q \rightarrow \infty} \chi_{i}\left(T_{m_{q}^{i}+1}^{0}\right)=l_{i} .
$$

Далее, по построению

$$
\varlimsup_{q \rightarrow \infty} \chi_{i}\left(T_{m_{q}^{i}}^{3}\right)=\varlimsup_{q \rightarrow \infty}\left(6 C \cdot 2^{m_{q}^{i}+1}-\sigma_{m_{q}^{i}}(\mu) T_{m_{q}^{i}}^{2}+l_{i} T_{m_{q}^{i}}^{0}\right) / T_{m_{q}^{i}}^{3}=\varlimsup_{q \rightarrow \infty} f_{q}^{i}(\mu)=f_{i}(\mu) .
$$

Окончательно получаем

$$
\lambda\left[x^{i}\right]=\max _{0 \leq j \leq 6} \max \left\{\varlimsup_{q \rightarrow \infty} \chi_{i}\left(T_{m_{q}^{i}}^{j}\right), \varlimsup_{q \rightarrow \infty} \chi_{i}\left(T_{m_{q}^{i}-1}^{j}\right)\right\}=\varlimsup_{q \rightarrow \infty} \chi_{i}\left(T_{m_{q}^{i}}^{3}\right)=f_{i}(\mu) .
$$

4.4. Покажем, что базис $\left(x^{1}(\cdot), \ldots, x^{n}(\cdot)\right)$ решений системы $\widetilde{C}(\cdot, \mu)$ является нормальным [17, 2.2]. Для этого докажем, что никакое подмножество набора решений $\left\{x^{1}(\cdot), \ldots, x^{n}(\cdot)\right\}$ не допускает понижающей комбинации $[17,2.3 .4]$, т. е. что для каждого $i \in\{2, \ldots, n\}$ и произвольного набора чисел $\alpha_{1}, \ldots, \alpha_{i}$, где $\alpha_{i} \neq 0$, выполняется равенство

$$
\lambda\left[\sum_{k=1}^{i} \alpha_{k} x^{k}\right]=\lambda\left[x^{i}\right] .
$$

Пусть $\mathrm{S}$ - какое-либо неограниченное подмножество временно́й полуоси $\mathbb{R}_{+}$. Тогда функционал

$$
\lambda\left[\left.x\right|_{\mathrm{S}}\right]=\varlimsup_{\mathrm{S} \ni t \rightarrow+\infty} \ln \|x(t)\|^{1 / t},
$$

определенный на линейном пространстве решений какой-либо системы (1), является показателем на этом пространстве в смысле [17, 2.1], а именно, обладает свойствами

$$
\begin{aligned}
\lambda\left[\left.(c x)\right|_{\mathrm{S}}\right] & =\lambda\left[\left.x\right|_{\mathrm{S}}\right] \text { при } c \neq 0, \\
\lambda\left[\left.(x+y)\right|_{\mathrm{S}}\right] & \leq \max \left\{\lambda\left[\left.x\right|_{\mathrm{S}}\right], \lambda\left[\left.y\right|_{\mathrm{S}}\right]\right\} .
\end{aligned}
$$

Положим $\mathrm{S}_{i}=\left\{T_{m_{q}^{i}}^{3}: q \in \mathbb{N}_{0}\right\}$. В силу п. 4.3 справедливы равенства

$$
\lambda\left[x^{i}\right]=\lambda\left[\left.x^{i}\right|_{\mathrm{S}_{i}}\right]=f_{i}(\mu), \quad \lambda\left[\left.x^{k}\right|_{\mathrm{S}_{i}}\right]=l_{k}, \quad k=\overline{1, i-1} .
$$

Рассмотрим сначала случай $f_{i}(\mu)=l_{i}$. Для любого решения $x(\cdot)$, удовлетворяющего условию $x_{i}(0) \neq 0$, из равенства (10) вытекает цепочка $\lambda[x] \geq \lambda\left[x_{i}\right] \geq l_{i}$. Следовательно, левая часть (11) не менее правой. Обратное неравенство вытекает из цепочки $f_{1}(\mu) \leq \ldots \leq f_{i}(\mu)$ и соотношений (12) при $\mathrm{S}=\mathbb{R}_{+}$.

Пусть теперь $f_{i}(\mu)>l_{i}$. Предположим, что равенство (11) не выполняется. Тогда из соотношений (12) при $\mathrm{S}=\mathbb{R}_{+}$получаем цепочку

$$
\lambda\left[\left.\left(\sum_{k=1}^{i} \alpha_{k} x^{k}\right)\right|_{\mathrm{S}_{i}}\right] \leq \lambda\left[\sum_{k=1}^{i} \alpha_{k} x^{k}\right]<\lambda\left[x^{i}\right]=\lambda\left[\left.x^{i}\right|_{\mathrm{S}_{i}}\right] .
$$

Применяя (12) при $\mathrm{S}=\mathrm{S}_{i}$, приходим к противоречию:

$$
\begin{gathered}
\lambda\left[\left.x^{i}\right|_{\mathrm{S}_{i}}\right]=\lambda\left[\left.\left(\sum_{k=1}^{i} \alpha_{k} x^{k}-\sum_{k=1}^{i-1} \alpha_{k} x^{k}\right)\right|_{\mathrm{S}_{i}}\right] \\
\leq \max \left\{\lambda\left[\left.\left(\sum_{k=1}^{i} \alpha_{k} x^{k}\right)\right|_{\mathrm{S}_{i}}\right], \lambda\left[\left.x^{1}\right|_{\mathrm{S}_{i}}\right], \ldots, \lambda\left[\left.x^{i-1}\right|_{\mathrm{S}_{i}}\right]\right\}<\lambda\left[\left.x^{i}\right|_{\mathrm{S}_{i}}\right] .
\end{gathered}
$$

В силу $[17,2.3 .10]$ базис $\left(x^{1}(\cdot), \ldots, x^{n}(\cdot)\right)$ является нормальным, а показатели его решений суть показатели системы $\widetilde{C}(\cdot, \mu)$. Таким образом, $\Lambda(\mu ; \widetilde{C})=F(\mu)$. 
4.5. Теперь вычислим показатели Ляпунова невозмущенной системы $\widetilde{A}$. По построению эта система диагональна, обозначим ее диагональные коэффициенты $p_{1}, \ldots, p_{n}$. В силу $[17,4.1]$ показатели Ляпунова системы $\widetilde{A}$ с точностью до перестановки совпадают с верхними интегральными средними значениями ее диагональных коэффициентов, т. е. с величинами

$$
\bar{p}_{i} \equiv \varlimsup_{t \rightarrow+\infty} \chi_{i}(t), \text { где } \chi_{i}(t)=t^{-1} \int_{0}^{t} p_{i}(\tau) d \tau, \quad t>0, \quad i=\overline{1, n} .
$$

Зафиксируем $i \in\{1, \ldots, n\}$. Поскольку на каждом полуинтервале $\Delta_{m}^{j}, m \in \mathbb{N}_{0}, j=\overline{1,6}$, функция $p_{i}$ постоянна, функция $\chi_{i}$ на каждом отрезке $\bar{\Delta}_{m}^{j}$ является дробно-линейной и, значит, монотонной (вообще говоря, нестрого). Следовательно, верхний предел в (13) можно вычислять по последовательности точек разрыва функции $p_{i}$, т. е. по концам тех отрезков $\bar{\Delta}_{m}^{j}$, $m \in \mathbb{N}_{0}, j=\overline{1,6}$, для которых хотя бы одно из чисел $m-i$ или $m-i+1$ делится на $n$. С помощью непосредственных вычислений нетрудно убедиться в справедливости равенства (10), а также соотношений

$$
\chi_{i}\left(T_{m_{q}^{i}}^{j}\right) \leq \chi_{i}\left(T_{m_{q}^{i}}^{0}\right)=l_{i}, \quad \chi_{i}\left(T_{m_{q}^{i}-1}^{j}\right) \leq \chi_{i}\left(T_{m_{q}^{i}-1}^{0}\right)=\chi_{i}\left(T_{m_{q}^{i}+1}^{0}\right)=l_{i}, \quad q \in \mathbb{N}_{0}, \quad j=\overline{1,5},
$$

из которых получаем, что $\bar{p}_{i}=l_{i}, i=\overline{1, n}$. Тогда из условия 1$)$ следует, что спектр показателей Ляпунова системы $\widetilde{A}$ совпадает с вектором $l$.

5. Построим теперь непрерывную матричнозначную функцию $A(\cdot)$ и семейство непрерывных матричнозначных функций $Q(\cdot, \mu), \mu \in M$, такие, что спектры показателей Ляпунова систем $\widetilde{A}$ и $A$, а также спектры показателей Ляпунова семейств $\widetilde{A}+\widetilde{Q}$ и $A+Q$ совпадают между собой и выполнены неравенства

$$
\|A(t)\| \leq\|\widetilde{A}(t)\|, \quad\|Q(t, \mu)\| \leq\|\widetilde{Q}(t, \mu)\|, \quad t \in \mathbb{R}_{+}, \quad \mu \in M .
$$

Положим $\delta_{k}=2^{-(k+1)} \exp \left(-\left(T_{k+1}\right)^{2}\right), k \in \mathbb{N}$. Для каждого $k \in \mathbb{N}$ выберем произвольную непрерывную (например, кусочно-линейную) функцию $s_{k}: \mathbb{R} \rightarrow[0,1]$, имеющую носитель, содержащийся в интервале $\left(T_{k}, T_{k+1}\right)$, и тождественно равную единице на отрезке $\left[T_{k}+\delta_{k}, T_{k+1}-\delta_{k+1}\right]$. Определим функцию $s: \mathbb{R}_{+} \rightarrow[0,1]$ равенством

$$
s(t)=\sum_{k=1}^{\infty} s_{k}(t), \quad t \in \mathbb{R}_{+} .
$$

Заметим, что носители слагаемых ряда (15) попарно не пересекаются, поэтому ряд (15) всюду сходится, а функция $s$ непрерывна, обращается в нуль в некоторой окрестности каждой из точек $T_{k}, k \in \mathbb{N}$, и тождественно равна единице на множестве $\bigcup_{k \in \mathbb{N}}\left[T_{k}+\delta_{k}, T_{k+1}-\delta_{k+1}\right]$.

Положим $A(t)=s(t) \widetilde{A}(t), Q(t, \mu)=s(t) \widetilde{Q}(t, \mu), C(t, \mu)=s(t) \widetilde{C}(t, \mu), t \in \mathbb{R}_{+}, \mu \in M$. Так как матричнозначная функция $\widetilde{A}(\cdot)$ постоянна на каждом из интервалов $\left(T_{k}, T_{k+1}\right), k \in \mathbb{N}_{0}$, то матричнозначная функция $A(\cdot)$ непрерывна. Проверим, что $Q \in \mathcal{E}^{n}[A](M)$. Свойство 3$)$ вытекает из оценок (9) и (14). Покажем, что матричнозначная функция $Q(\cdot, \cdot)$ непрерывна. Пусть заданы $t_{0} \in \mathbb{R}_{+}$и $\mu_{0} \in M$. Если $t_{0}$ совпадает с одной из точек $T_{k}, k \in \mathbb{N}$, или лежит внутри одного из промежутков $\Delta_{m}^{j}, m \in \mathbb{N}_{0}, j=1,3,4,6$, то по построению найдется такая окрестность $U$ точки $t_{0}$, что $Q(t, \mu)$ - нулевая матрица при всех $t \in U$ и $\mu \in M$. Если $t_{0}$ лежит внутри одного из промежутков $\Delta_{m}^{j}, m \in \mathbb{N}_{0}, j=2,5$, то $Q(\cdot, \cdot)$ непрерывна в точке $\left(t_{0}, \mu_{0}\right)$ как произведение постоянной по $t$ в некоторой окрестности точки $t_{0}$ и непрерывной по $\mu$ матричнозначной функции $\widetilde{Q}(\cdot, \cdot)$ и непрерывной функции $s(\cdot)$.

Заметим, что по построению $\|\widetilde{C}(t, \mu)\| \leq 12 C, t \in \mathbb{R}_{+}, \mu \in M$. Пусть для некоторого $l \in \mathbb{N}$ выполнено включение $t \in\left[T_{l}, T_{l+1}\right)$. Тогда справедлива цепочка неравенств

$$
\int_{T_{1}}^{t}\|s(\tau) \widetilde{C}(\tau, \mu)-\widetilde{C}(\tau, \mu)\| e^{\tau^{2}} d \tau \leq 12 C \sum_{k=1}^{l+1} 2 \delta_{k} \exp \left(\left(T_{k+1}\right)^{2}\right) \leq 12 C,
$$


из которой получаем, что при всех $\mu \in M$ интеграл $\int_{0}^{+\infty}\|C(\tau, \mu)-\widetilde{C}(\tau, \mu)\| e^{\tau^{2}} d \tau$ сходится. Следовательно [17, теорема 29.1.1], спектры показателей Ляпунова систем $C(\cdot, \mu)$ и $\widetilde{C}(\cdot, \mu)$ совпадают между собой. Путем аналогичных рассуждений устанавливается совпадение спектров систем $A$ и $\widetilde{A}$.

6. Пусть теперь заданы произвольные набор $l \in \mathbb{R}^{n}$ и вектор-функция $F: M \rightarrow \mathbb{R}^{n}$, удовлетворяющие условиям 1)-4) теоремы. По доказанному существуют система $A_{0} \in \mathcal{M}_{n}$ и семейство $Q \in \mathcal{E}_{n}\left[A_{0}\right](M)$, удовлетворяющие равенствам $\Lambda\left(A_{0}\right)=\left(0, l_{2}-l_{1}, \ldots, l_{n}-l_{1}\right)$ и $\Lambda\left(\cdot ; A_{0}+Q\right)=\left(f_{1}-l_{1}, \ldots, f_{n}-l_{1}\right)$. Воспользуемся следующим хорошо известным утверждением: показатели Ляпунова $\lambda_{i}(B)$ и $\lambda_{i}\left(B+a E_{n}\right), i=\overline{1, n}$, систем

$$
\dot{x}=B(t) x \quad \text { и } \quad \dot{y}=\left(B(t)+a E_{n}\right) y, \quad x, y \in \mathbb{R}^{n}, \quad t \in \mathbb{R}_{+},
$$

соответственно, где $a \in \mathbb{R}$ фиксировано, а $E_{n}$ - единичная $(n \times n)$-матрица, связаны равенством $\lambda_{i}\left(B+a E_{n}\right)=\lambda_{i}(B)+a$, которое вытекает из того, что для решений $x(\cdot)$ и $y(\cdot)$ этих систем с одним и тем же начальным вектором $(x(0)=y(0))$ имеет место тождество $y(t) \equiv x(t) \exp (a t)$, $t \in \mathbb{R}_{+}$.

Положим $A(t)=A_{0}(t)+l_{1} E_{n}, t \in \mathbb{R}_{+}$. Тогда по выбору системы $A_{0}$ и вследствие сказанного выше получаем, что $\Lambda(A)=l$. По той же причине спектр $\Lambda(\cdot ; A+Q)$ показателей Ляпунова семейства $A+Q$ с так определенной матрицей $A(\cdot)$ совпадает с вектор-функцией $F(\cdot)$. Условие $Q \in \mathcal{E}_{n}[A](M)$ также, очевидно, выполняется.

Теорема доказана.

Д о к а з а т е л ь с т в о следствия 1. В рассматриваемом случае непрерывные функции $f_{m}^{i}, m \in \mathbb{N}, i=\overline{1, n}$, в представлении (4) можно без ограничения общности считать многочленами. Действительно, по теореме Вейерштрасса для любых $m \in \mathbb{N}$ и $i=\overline{1, n}$ найдется многочлен $p_{m}^{i}$ такой, что $f_{m}^{i}(\mu)-1 / m<p_{m}^{i}(\mu)<f_{m}^{i}(\mu)+1 / m$ при всех $\mu \in M$. Следовательно, справедливо равенство

$$
f_{i}(\mu)=\varlimsup_{m \rightarrow \infty} p_{m}^{i}(\mu), \quad \mu \in M, \quad i=\overline{1, n} .
$$

Тогда для каждого $m \in \mathbb{N}$ функция $\sigma_{m}$ из п. 2 доказательства теоремы является многочленом, а коэффициенты матричнозначной функции $\widetilde{Q}(t, \cdot)$, а значит, и функции $Q(t, \cdot)$ при всяком $t \in \mathbb{R}_{+}$являются аналитическими функциями параметра.

Следствие доказано.

Д о к а з а т е л ь с т в о следствия 2. Установим необходимость указанных условий. Условие а) вытекает из определения класса $\mathcal{E}_{n}[A](M)$, поскольку для любых $B \in \mathcal{M}_{n}$ и $k=\overline{0, n-1}$ неравенства es $(B) \leq k$ и $\lambda_{k+1}(B) \geq 0$ равносильны. Условие б) равносильно требованию, что для каждого $k=\overline{0, n-1}$ множество $\{\mu \in M \mid \operatorname{es}(\cdot ; A+Q) \leq k\}$ является $G_{\delta}$-множеством. Последнее вытекает из равенства

$$
\{\mu \in M \mid \mathrm{es}(\cdot ; A+Q) \leq k\}=\left\{\mu \in M \mid \lambda_{k+1}(\cdot ; A+Q) \geq 0\right\}
$$

и утверждения теоремы.

Докажем достаточностъ. Пусть заданы $d \in \mathcal{Z}_{n}$ и функция $f: M \rightarrow \mathcal{Z}_{n}$, удовлетворяющие условиям а) и б). Тогда для любого $r \in \mathbb{R}$ множество $\{\mu \mid f(\mu) \leq r\}$ является $G_{\delta^{-}}$ множеством. Следовательно, тем же свойством обладает при всех $k=\overline{1, n}$ и множество $U_{k}=$ $\{\mu \in M \mid f(\mu)<k\}$, так как при всех $\mu \in M$ неравенство $f(\mu)<k$ равносильно неравенству $f(\mu) \leq k-1$. Для каждого $k=\overline{1, n}$ определим функцию $f_{k}: M \rightarrow\{-1,0\}$ равенствами: $f_{k}(\mu)=0$, если $\mu \in U_{k}$, и $f_{k}(\mu)=-1$ в противном случае. Несложно видеть, что $f_{k}-$ функция класса $\left({ }^{*}, G_{\delta}\right)$. В самом деле, множество $\left\{\mu \in M \mid f_{k}(\mu) \geq r\right]$ пусто, если $r>0$, совпадает с множеством $U_{k}$, если $r \in(-1,0]$, и совпадает со всем пространством $M$, если $r \leq-1$. 
Далее, из цепочки включений $U_{1} \subset U_{2} \subset \ldots \subset U_{n}$ следует цепочка неравенств $f_{1} \leq \ldots \leq f_{n}$. Кроме того, по построению для любого $k=\overline{1, n}$ неравенство $f_{k}(\mu)=-1$ равносильно неравенству $f(\mu) \geq k$, откуда получаем $d \geq k$. Следовательно, в силу теоремы существуют система $A \in \mathcal{M}_{n}$ и семейство $Q \in \mathcal{E}^{n}[A](M)$ такие, что первые $d$ показателей Ляпунова системы $A$ равны -1 , остальные равны нулю и спектр показателей Ляпунова $\left(\lambda_{1}(\cdot ; A+Q), \ldots, \lambda_{n}(\cdot ; A+Q)\right)$ семейства $A+Q$ совпадает с вектор-функцией $\left(f_{1}, \ldots, f_{n}\right)$. Следующие цепочки эквивалентностей

$$
f(\mu)=n \Leftrightarrow \mu \notin U_{n} \Leftrightarrow \lambda_{n}(\mu ; A+Q)<0 \Leftrightarrow \operatorname{es}(\mu ; A+Q)=n, \quad \mu \in M,
$$

$f(\mu)=k \Leftrightarrow \mu \in U_{k+1} \backslash U_{k} \Leftrightarrow \lambda_{k}(\mu ; A+Q)<0 \leq \lambda_{k+1}(\mu ; A+Q) \Leftrightarrow \operatorname{es}(\mu ; A+Q)=k, \quad k=\overline{1, n-1}$,

показывают, что функции $f(\cdot)$ и es $(\cdot ; A+Q)$ совпадают, как только одна из них принимает значение из множества $\{1, \ldots, n\}$. Но тогда и значение "нуль" они принимают одновременно. Таким образом, $f(\mu)=\operatorname{es}(\mu ; A+Q)$ при всех $\mu \in M$.

Следствие доказано.

\section{СПИСОК ЛИТЕРАТУРЫ}

1. Ляпунов А.М. Собрание сочинений: в 6 т. Т. 2. Общая задача об устойчивости движения. М.; Л.: Изд-во АН СССР, 1956. 476 с.

2. Демидович Б.П. Лекции по математической теории устойчивости. М.: Наука, 1967. 472 с.

3. Perron O. Die Ordnungszahlen linearer Differentialgleichungssysteme // Math. Zeitschr. 1930. Vol 31, iss. 4. P. $748-766$.

4. Perron O. Die Stabilitätsfrage bei Differentialgleichungen // Math. Zeitschr. 1930. Vol 32, iss. 5. P. 703-728.

5. Леонов Г.А. Хаотическая динамика и классическая теория устойчивости движения. М.; Ижевск: Регулярная и хаотическая динамика, 2006. 168 с.

6. Коровин С.К., Изобов Н.А. Реализация эффекта Перрона смены значений характеристических показателей решений дифференциальных систем // Дифференц. уравнения. 2010. Т. 46, № 11. C. $1536-1550$.

7. Барабанов Е.А., Быков В.В., Карпук М.В. Полное описание спектров показателей Ляпунова линейных дифференциальных систем, непрерывно зависящих от параметра равномерно на временно́й полуоси // Дифференц. уравнения. 2018. Т. 54, № 12. С. 1579-1588. doi: $10.1134 / \mathrm{S} 0374064118120014$.

8. Миллионщиков В.М. Бэровские классы функций и показатели Ляпунова. I // Дифференц. уравнения. 1980. Т. 16, № 8. С. 1408-1416.

9. Бэр Р. Теория разрывных функций М.; Л.: ГТТИ, 1932. 134 с.

10. Хаусдорф Ф. Теория множеств. М.; Л.: ОНТИ, 193. 306 с.

11. Барабанов Е.А., Быков В.В. Описание линейного эффекта Перрона при параметрических возмущениях, убывающих к нулю на бесконечности // Устойчивость, управление, дифференциальные игры (SCDG2019): материалы Междунар. конф., посвященной 95-летию со дня рождения академика Н.Н. Красовского / ИММ УрО РАН. Екатеринбург, 2019. С. 48-53.

12. Миллионщиков В.М. Показатели Ляпунова как функции параметра // Мат. сб. 1988. Т. 137, № 3. С. $364-380$.

13. Быков В.В. Функции, определяемые показателями Ляпунова семейств линейных дифференциальных систем, непрерывно зависящих от параметра равномерно на полуоси // Дифференц. уравнения. 2017. Т. 53, № 12. С. 1579-1592. doi: 10.1134/S0374064117120019.

14. Stepanoff W. Sur les suites des fonctions continues // Fund. Math. 1928. Vol. 11. pp. 264-274.

15. Карпук М.В. Показатели Ляпунова семейств морфизмов метризованных векторных расслоений как функции на базе расслоения // Дифференц. уравнения. 2014. Т. 50, № 10. С. 1332-1338. doi: $10.1134 / \mathrm{S} 0374064114100057$. 
16. Изобов Н.А. Введение в теорию показателей Ляпунова. Минск.: Изд-во Белорус. гос. ун-т, 2006. 319 c.

17. Былов Б.Ф., Виноград Р.Э., Гробман Д.М., Немыцкий В.В. Теория показателей Ляпунова и ее приложения к вопросам устойчивости. М.: Наука, 1966. 576 с.

Поступила 30.09.2019

После доработки 8.11.2019

Барабанов Евгений Александрович

Принята к публикации 11.11.2019

канд. физ.-мат. наук, ведущий науч. сотрудник

Институт математики НАН Беларуси

г. Минск

e-mail: bar@im.bas-net.by

Быков Владимир Владиславович

канд. физ.-мат. наук, доцент

МГУ имени М.В. Ломоносова

г. Москва

e-mail: vvbykov@gmail.com

\section{REFERENCES}

1. Lyapunov A.M. The general problem of the stability of motion. Int. J. Control, 1992, vol. 55, no. 3, pp. 531-773.

2. Demidovich B.P. Lektsii po matematicheskoi teorii ustoichivosti [Lectures on mathematical stability theory]. Moscow: Nauka Publ., 1967, 472 p.

3. Perron O. Die Ordnungszahlen linearer Differentialgleichungssysteme. Math Z., 1930, vol. 31, no. 1, pp. 748-766. doi: 10.1007/BF01246445 .

4. Perron O. Die Stabilitätsfrage bei Differentialgleichungen. Math Z., vol. 32, no. 1, pp. 703-728. doi: 10.1007/BF01194662 .

5. Leonov G.A. Khaoticheskaya dinamika $i$ klassicheskaya teoriya ustoichivosti dvizheniya [Chaotic dynamics and classical theory of motion stability]. Izhevsk: "Reguljarnaja i haoticheskaja dinamika" Publ., 2006, 168 p. ISBN: 5-93972-470-1.

6. Korovin S.K., Izobov N.A. Realization of the Perron effect whereby the characteristic exponents of solutions of differential systems change their values. Diff. Equat., vol. 46, no. 11, pp. 1537-1551. doi: 10.1134/S0012266110110029.

7. Barabanov E.A., Bykov V.V., Karpuk M.V. Complete description of the Lyapunov spectra of families of linear differential systems whose dependence on the parameter is continuous uniformly on the time semiaxis. Diff. Equat., 2018, vol. 54, no. 12, pp. 1535-1544. doi: 10.1134/S0012266118120017.

8. Millionshchikov V.M. Baire classes of functions and Lyapunov exponents. I. Diff. Uravneniya, 1980, vol. 16, no. 8, pp. 1408-1416 (in Russian).

9. Baire R. Teoriya razryvnykh funktsii [Theory of discontinuous functions]. Moscow; Leningrad: GTTI Publ., 1932, 134 p. ISBN: 978-5-4460-6297-3 . Original French text published in Baire R. Leçons sur les Fonctions Discontinues, Paris: Gauthier-Villars, 1905, 127 p.

10. Hausdorff F. Set theory. N Y: Chelsea Publ. Company, 1962, 352 p. Translated to Russian under the title Teoriya mnozhestv. Moscow; Leningrad: ONTI Publ., 1937, 306 p.

11. Barabanov E.A., Bykov V.V. Description of a linear Perron effect under parametric perturbations vanishing at infinity. In: T.F. Filippova, V.I. Maksimov, A.M. Tarasyev (eds.), Stability, Control, Differential Games (SCDG2019): Proc. Internat. Conf. devoted to the 95th anniversary of Academician N.N. Krasovskii (Yekaterinburg, Russia, 16-20 September 2019). Yekaterinburg, 2019, pp. 48-53 (in Russian).

12. Millionshchikov V.M. Lyapunov exponents as functions of a parameter. Math. USSR-Sb., 1990, vol. 65, no. 2, pp. 369-384. doi: 10.1070/SM1990v065n02ABEH001147.

13. Bykov V.V. Functions determined by the Lyapunov exponents of families of linear differential systems continuously depending on the parameter uniformly on the Half-Line. Diff. Eq., 2017, vol. 53, no. 12, pp. 1529-1542. doi: 10.1134/S0012266117120011. 
14. Stepanoff W. Sur les suites des fonctions continues. Fund. Math., 1928, vol. 11, no. 1, pp. 264-274.

15. Karpuk M.V. Lyapunov exponents of families of morphisms of metrized vector bundles as functions on the base of the bundle. Diff. Eq., 2014, vol. 50, no. 10, pp. 1322-1328. doi: 10.1134/S001226611410005X.

16. Izobov N.A. Lyapunov exponents and stability. Cambridge: Cambridge Scientific Publ., 2012,353 p. ISBN: 978-1-908106-25-4 . Original Russian text published in Izobov N.A. Vvedenie v teoriyu pokazatelei Lyapunova. Minsk: Belorusskij Gosudarstvennyj Universitet Publ., 2006, 319 p.

17. Bylov B.F., Vinograd R.E., Grobman D.M., Nemytskii V.V. Teoriya pokazatelei Lyapunova $i$ ee prilozheniya $k$ voprosam ustoichivosti [Theory of Lyapunov exponents and its application to stability problems]. Moscow: Nauka Publ., 1966, 576 p.

Received September 30, 2019

Revised November 8, 2019

Accepted November 11, 2019

Evgenii Aleksandrovich Barabanov, Cand. Sci. (Phys.-Math.), Institute of Mathematics of National Academy of Sciences, Minsk, 119991 Belarus, e-mail: bar@im.bas-net.by .

Vladimir Vladislavovich Bykov, Cand. Sci. (Phys.-Math.), Lomonosov Moscow State University, Moscow, 119991 Russia, e-mail: vvbykov@gmail.com .

Cite this article as: E. A. Barabanov, V.V.Bykov. Description of the linear Perron effect under parametric perturbations exponentially vanishing at infinity, Trudy Instituta Matematiki i Mekhaniki URO RAN, 2019, vol. 25, no. 4, pp. 31-43. 